\title{
Aplicación del método de opciones reales en la valoración de proyectos inmobiliarios
}

Fecha de entrega: 7 de junio 2013

Fecha de aceptación: 28 de noviembre 2013

\section{Eric Forcael ${ }^{1}$, Alejandro Andalaft ${ }^{2}$, Roberto Schovelin ${ }^{3}$ y Pablo Vargas ${ }^{3}$}

${ }^{1}$ Departamento de Ingeniería Civil y Ambiental, Universidad del Bío-Bío, Avenida Collao 1202, Concepción, Chile, eforcael@ubiobio.cl

${ }^{2}$ Departamento de Ingeniería Industrial, Universidad de Concepción, Edmundo Larenas s/n, Concepción, Chile, aandalaf@udec.cl

${ }^{3}$ Departamento de Ingeniería Civil Industrial, Universidad del Bío-Bío, Avenida Collao 1202, Concepción, Chile, rschovel@ubiobio.cl,pabvarga@alumnos.ubiobio.cl

En este trabajo se aplica la metodología de las opciones reales a un proyecto inmobiliario absorbiendo la variabilidad que puede presentar. Al mismo tiempo se complementa con la evaluación tradicional del VAN que se utiliza en este tipo de proyectos por las empresas del sector inmobiliario, el cual indica en cuánto aumentará de valor la empresa si se realiza el proyecto. Sin embargo, esta evaluación económica considera un único escenario esperado, asumiendo una gestión estática en el desarrollo del proyecto. La teoría del análisis de inversiones ha incorporado de la teoría de valoración de opciones financieras la valoración de oportunidades de inversión en un escenario flexible y de incertidumbre. Conocida como teoría de las opciones reales, nace como complemento a la evaluación tradicional de proyectos. Con el fin de aplicar el método propuesto se ha considerado el estudio de un proyecto inmobiliario de casas ubicado en el Gran Concepción, casas en el rango de UF1000 a UF1500. Este proyecto se evaluó en una primera instancia mediante la metodología tradicional para posteriormente evaluarlo de acuerdo al método de las opciones reales; a través de un modelo Binomial Logarítmico Transformado. Se comprobó que el valor del proyecto en estudio aumentó significativamente en relación a la evaluación tradicional al valorar las opciones reales que comúnmente no se consideran en este tipo de proyecto; abandonar y expandir. Por consiguiente, la metodología de valoración mediante opciones reales, manifiesta el potencial que posee un proyecto cuando presenta incertidumbre, utilizando ésta como un elemento fundamental en la agregación de valor al mismo.

Palabras clave: VAN, incertidumbre, opciones reales, proyecto inmobiliario
In this work the real options methodology is applied to an estate project taking into account the project variability. At the same time the traditional Net Present Value NPV evaluation is carried out since it is what the estate sector uses normally in this type of projects. The NPV represents how much will increase the value of a company if the project is carried out. However, this economic evaluation considers only one possible scenario expected, assuming a fixed management during the whole project life. The investment analysis theory has included the investment opportunities valuation from the financial options valuation theory within a flexible and uncertain environment. This is known as the theory of real valuation options, which arises as a complement to the traditional project evaluation methods. With the aim to apply this proposed method, an estate project of houses located in the Great Concepcion is considered with houses in the price range of UF1000 up to UF1500. This project was first evaluated through the traditional methodology and later on it was evaluated using the real options method, applying the transformed logarithm binomial model. It was verified that the project value increased significantly in relation to the traditional evaluation since real options were included such as abandonment and expansion. Therefore, the evaluation methodology by means of real options shows the potential that a project can have when uncertainty is prevalent, which is incorporated in the adding value to the project. 


\section{Introducción}

En Chile, el tratamiento de oportunidades de inversión conocida como método de las opciones reales, ha tenido un gran desarrollo en la evaluación de proyectos del sector forestal, I+D, energético, mineros, ambiental y en proyectos socioeconómicos. No así en el sector inmobiliario, en donde aún no ha sido aplicada. Por esta razón, en esta investigación, se realizará la aplicación de las opciones reales en el sector inmobiliario del Gran Concepción, específicamente en construcciones de casas, obteniendo el valor de las opciones que comúnmente se ven presentes en este tipo de proyectos: abandonar, esperar y ver, expandir y crecer por etapas, utilizando el Modelo Binomial Logarítmico Transformado MBLT para obtener la valoración de las opciones. Con estos resultados, se pretende mostrar que el valor de un proyecto no depende únicamente de sus flujos de caja directamente atribuidos, sino que también de las oportunidades y la flexibilidad con que se cuenta para responder ante la incertidumbre.

Evaluar un proyecto de inversión del sector inmobiliario, específicamente en la construcción de viviendas, puede tornarse complejo debido a la incertidumbre de acontecimientos que pueden surgir durante su desarrollo: cambios en la demanda, la oferta, los precios, los costos, entre otros. Así entonces, se requiere incorporar la flexibilidad en la toma de decisiones, y la capacidad de proponer comportamientos estratégicos en los procesos de inversión, sobre todo en el mercado inmobiliario que fácilmente se ve afectado cuando el mercado es incierto y/o cambiante.

Complementar la evaluación tradicional de proyectos inmobiliarios con la teoría de las opciones reales, llevaría a generar una flexibilidad operativa capaz de adaptar las acciones futuras en un proyecto a los cambios que éste se vería enfrentado, debido a las condiciones del mercado, obligando a las inmobiliarias a considerar las opciones más comunes en sus proyectos: aplazar, crecer por etapas o abandonar.

La teoría de las opciones reales, proporciona un método para analizar las decisiones de tipo estratégicas en condiciones de incertidumbre, ya que incorporan la flexibilidad como una herramienta de decisión y en donde se pueden realizar una revisión de ésta durante el desarrollo de un proyecto, permitiendo así no seguir un plan establecido, sino adaptarse a los acontecimientos futuros (Amram y Kulatilaka, 2000).

\section{Estudios previos}

Concerniente a la línea de investigación seguida por este artículo, algunos autores han abordado la problemática de la evaluación de los proyectos inmobiliarios y de construcción considerando la Teoría de las Opciones Reales. Titman (1985) analiza los precios de los terrenos urbanos en un contexto de incertidumbre y su repercusión sobre los proyectos de construcción. La decisión de construir va a sopesar, por un lado, los costos de oportunidad asociados a mantener la tierra inactiva en espera y, por otro, las ganancias esperadas de construir un proyecto más apropiado en un momento futuro. También en el análisis de terrenos, Quigg (1993) analiza un modelo de valoración de opciones reales, que contiene la opción de esperar para desarrollar un terreno urbano, donde se analiza el efecto de la volatilidad sobre las decisiones empresariales y su efecto positivo sobre el valor del proyecto de desarrollo en función del terreno. De manera similar, en aquellos casos en los cuales se busca determinar la explotación de un terreno por etapas, Cappoza y Li (1994) determinaron el valor de terrenos y el momento óptimo para construir, demostrando cómo influye el valor del proyecto al aumentar los valores óptimos invirtiendo por etapas.

Por su parte, Majd y Pindyck (1986) analizan el momento óptimo de tiempo para desarrollar un proyecto de construcción, valorando las opciones inherentes al mismo y teniendo en cuenta la flexibilidad empresarial a la hora de tomar decisiones. Estos autores determinaron que la decisión de inversión es muy sensible al nivel de riesgo, incluso más que la sensibilidad sugerida por los modelos de inversión tradicionales; sensibilidad debida sobre todo a la flexibilidad en las decisiones empresariales.

Así también, Williams (1991) analiza el valor de las opciones, considerando por una parte el desarrollo de un proyecto habitacional, contra la posibilidad de no realizar dicho proyecto. En este caso, el inversionista elige el momento óptimo para desarrollar el proyecto en cuestión, donde la opción es más valiosa cuanto más inciertos sean los cambios sobre los ingresos y los costos de construcción en el tiempo.

Específicamente en relación a proyectos de construcción, es común enfrentar los problemas de infraestructura (vial, sanitaria, etc.). A este respecto, Hinojosa (2008) incorpora 
las Opciones Reales en la valoración de infraestructura sanitaria, ante una posible expansión del suministro de agua potable, considerando aspectos políticos relevantes y el comportamiento de las economías emergentes.

Finalmente, Calle y Tamayo (2009) caracterizan la realización de un proyecto de urbanización, que al considerar las perspectivas de crecimiento del sector de la construcción, plantearon la aplicación de la teoría basándose en dos opciones reales: abandonar el proyecto una vez iniciado si las condiciones así lo requerían, o esperar un mejor momento para comenzar a construir.

No obstante lo anterior, dentro de la industria inmobiliaria y de la construcción, existe limitada evidencia sobre la existencia de estudios comparativos entre distintos métodos de evaluación de proyectos con carácter inmobiliario, que es en definitiva el objetivo principal de esta investigación.

\section{Las opciones reales}

Los métodos tradicionales de evaluación de proyectos como VAN y TIR y el pensamiento estratégico, son presentados como dos enfoques complementarios en la evaluación de proyectos de inversión. Ambos enfoques se pueden considerar como dos posturas que pueden llevar a recomendaciones opuestas, ya que sus principios de aplicación no están relacionados entre sí.

Al momento de tomar decisiones, se pueden desestimar los resultados de tipo cuantitativo, por lo cual se anteponen factores estratégicos y de flexibilidad operativa, los cuales se considerarían de igual o superior valor que los flujos de caja obtenidos por la teoría financiera.

La flexibilidad operativa a la que los proyectos se ven afectados o beneficiados durante su ejecución, en respuesta a movimientos inesperados del mercado, no es capturada adecuadamente por los métodos tradicionales de evaluación económica, ya que estos consideran un único escenario esperado, por lo que se debe asumir una gestión estática en el desarrollo del mismo; apoyados a una única estrategia operativa. Por ende, es propicio considerar una flexibilidad administrativa, como elemento fundamental a la hora de evaluar, que sea capaz de adaptarse a las acciones futuras ocasionadas por los cambios del mercado (Trigeorgis, 1996).

Por esta razón nace la idea de la teoría de las opciones reales, como una herramienta complementaria en la evaluación de proyectos, ya que considera métodos que permiten implementar y evaluar el componente estratégico de los proyectos de manera sistemática y metódica, además que es capaz de utilizar la información concerniente a los mercados financieros. Cambian la manera de tratar las decisiones de tipo estratégicas en condiciones de incertidumbre, incorporando la flexibilidad como una herramienta de decisión, permitiendo hacer una revisión de ésta durante la ejecución del proyecto, adaptándose a los acontecimientos futuros, ocasionados comúnmente por el mercado.

En este sentido Trigeorgis y Mason (1987) mencionan que las opciones reales representan la flexibilidad de un proyecto y son la herramienta de decisión que permite hacer una revisión en forma continua respecto de las decisiones en el desarrollo del mismo, permitiendo, así, no seguir un plan establecido, adaptándose a los eventos futuros.

Por su parte, Amram y Kulatilaka (2000) definieron el método de opciones reales como la extensión de la teoría de opciones financieras a las opciones sobre activos reales (no financieros). Mientras que las opciones financieras se detallan en el contrato, las opciones reales objeto de inversiones estratégicas, deben ser identificadas y especificadas. El paso de las opciones financieras a las opciones reales, requiere una filosofía determinada, una forma de ver las cosas que introduzca la disciplina de los mercados financieros en las decisiones internas de la inversión estratégica. Además, hacen mención de que desde una mirada tradicional, cuando mayor sea el nivel de incertidumbre, menor es el valor del activo. Del punto de vista de las opciones reales, demuestra que una mayor incertidumbre puede provocar un valor superior del activo, si los ejecutivos logran identificar y utilizar sus opciones para responder con flexibilidad al desarrollo de los acontecimientos.

Por lo mencionado anteriormente, las oportunidades de aumentar valor pueden crearse en la medida que exista incertidumbre. En la Figura 1 se muestra que a medida que la incertidumbre va incrementando esto conduciría a un posible incremento en el valor de los activos, siempre y cuando los ejecutivos identifiquen y utilicen sus opciones, con el propósito de responder flexiblemente en sus actividades. Por lo tanto, ahí se muestra uno de los 
más importantes cambios en el enfoque de las opciones reales: La incertidumbre crea oportunidades (Amram y Kulatilaka, 2000).

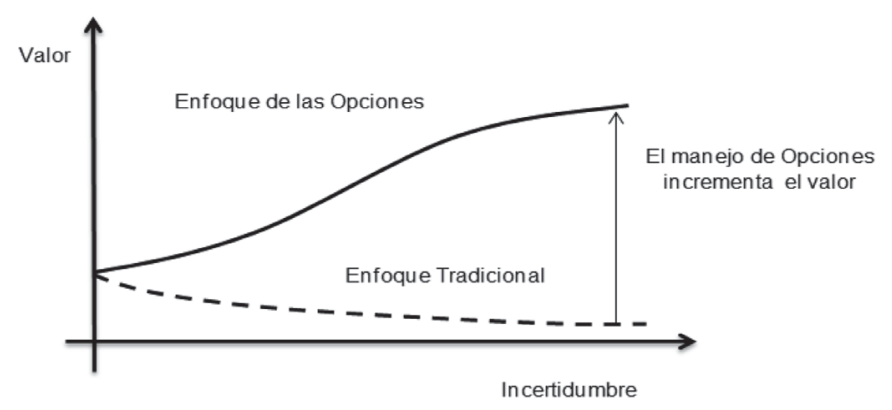

Figura 1: Enfoque de las Opciones Reales: La Incertidumbre incrementa el valor (adaptado de Amram y Kuatilaka, 2000)

La metodología de las Opciones Reales es utilizada como complemento de las técnicas tradicionales de valoración (Sánchez y Andalaft, 2010), con el fin de evaluar desde el punto de vista económico, inversiones o activos cuyos flujos de caja se tornan inciertos, otorgando ventajas de evaluar proyectos de inversión, tales como:

- $\quad$ Proporcionan el valor del proyecto sin la necesidad de utilizar métodos en los cuales varían la tasa de descuento, ya que las opciones reales utilizan una única tasa de descuento dada por el inversionista.

- $\quad$ Permite incluir flexibilidades operativas durante el ciclo de vida del proyecto (abandono, expansión, contracción, cambios estratégicos)

- $\quad$ Entrega como subproducto la política de operación que maximiza el valor del proyecto a través del tiempo.

\section{Metodología y desarrollo del problema Métodos tradicionales de evaluación y las opciones reales}

Dentro de los métodos tradicionales de evaluación de proyectos, el más utilizado por las empresas del sector inmobiliario es el Método del Flujo de Caja Descontado FCD, con el cual se determina el Valor Presente Neto o VAN del proyecto. Mediante el VAN se puede estimar si es conveniente invertir y si el proyecto vale más de lo que cuesta, al calcular cuánto valdrían sus flujos de caja si el derecho sobre ellos se ofreciera a los inversionistas independientemente del proyecto, y si se negociara en los mercado de capitales (Brealey et al., 2007). Sapag y Sapag (2008) señalan que el flujo de caja de cualquier proyecto se compone de cuatro elementos básicos: a) los egresos iniciales de fondos, b) los ingresos y egresos de operación, c) el momento en que incurren estos ingresos y egresos, y d) el valor de desecho o salvamento del proyecto.

Por otra parte, Fontaine (1997) afirma que la inversión será rentable sólo si el valor actual de los flujos de beneficios netos que genera es positivo, descontando estos flujos a la tasa de descuento pertinente para el inversionista. Este valor actual mide, en moneda de hoy, cuánto más rico es el inversionista por invertir en el proyecto, en lugar de hacerlo en función de la alternativa que rinde la tasa de descuento. En ocasiones las técnicas de evaluación tradicional, como el VAN, no logran satisfacer completamente a los evaluadores ya que, por lo general, cuando se trata de proyectos inmobiliarios, no se basan en estudios matemáticos o financieros, sino más bien en la experiencia; comienzan a evaluar posibles opciones en la realización de sus proyectos: abandonar, aplazar, crecer por etapas o expandir. Esto se explica, dado que al momento de tomar decisiones es posible desestimar los resultados de tipo cuantitativo, anteponiendo factores estratégicos y de flexibilidad operativa, por lo que el inversionista valora más los factores estratégicos de tipo cualitativo que los factores cuantitativos del flujo de caja al momento de tomar una decisión.

Debido a lo anterior, Campa (1994) señala que la evaluación tradicional de proyectos presenta algunas limitaciones, ya que las inversiones se encuentran en ambientes de elevada incertidumbre. La metodología tradicional considera un único escenario esperado de flujos de caja, por lo que se asume una gestión estática durante el desarrollo del proyecto.

Por otro lado, la metodología de opciones reales, permite complementar la evaluación tradicional, ya que considera la implementación y evaluación del componente estratégico de los proyectos de manera sistemática y metódica. De esta forma, las opciones reales cambian la manera de enfrentar las decisiones de tipo estratégicas en condiciones de incertidumbre, incorporando la flexibilidad como una herramienta de decisión, permitiendo hacer una revisión 
de ésta durante la ejecución del proyecto, adaptándose a los acontecimientos futuros; comúnmente presentes en el mercado.

Lo anterior se explica adicionando al Valor Presente Neto tradicional del proyecto, el valor de las opciones implícitas en el proyecto; dando como resultado el concepto de Valor Actual Neto Expandido o Estratégico (Mascareñas, 2003), mostrado en la ecuación (1).

VAN Expandido = VAN Tradicional + Valor de las
Opciones Reales

Así entonces, es posible plantear la valoración de proyectos inmobiliarios teniendo presente las oportunidades de inversión como opciones reales (opciones sobre bienes o activos reales).

\section{Opciones reales presentes en proyectos inmobiliarios}

La teoría de las opciones reales estudia la forma de valorar un proyecto de inversión empresarial de forma análoga a la que se utiliza para valorar una opción financiera. Para el caso del sector inmobiliario, por ejemplo en la construcción de viviendas o departamentos, se estará ante una opción de compra, en la que se paga un precio de ejercicio (inversión inicial), para tener derecho a ejercer dicha opción del activo subyacente (realización del proyecto de construcción) en el futuro. Dado que la inversión en el sector inmobiliario puede ser realizada en cualquier momento en que se desee, se ha de considerar para el desarrollo de esta investigación la opción americana, que a diferencia de las opciones de compra europeas, el momento en que pueden ser ejercitadas está prefijado (Lamothe, 1995).

El caso de estudio está emplazado en el Gran Concepción (contempla las comunas de Concepción, San Pedro de la Paz, Chiguayante, Penco, Hualpén y Talcahuano), lugar para el cual la Figura 2 evidencia que para este conglomerado urbano, durante el tercer trimestre de 2012, fueron 456 los departamentos vendidos, un 30\% más que lo comercializado en similar periodo anterior, y un $15 \%$ más que el último trimestre del año anterior. Respecto a la venta de casas, durante el primer trimestre del año 2012, las unidades comercializadas fueron 378 , un $20 \%$ menos que similar periodo del año anterior; sin embargo, un 35\% menos que el trimestre inmediatamente anterior, por lo que se muestra un mercado variable, sujeto a cambios bruscos entre un período y otro. Así, frente a la incertidumbre que evidencia el sector inmobiliario de la zona en estudio, es apropiado considerar que las inmobiliarias planteen tres opciones reales para evaluar sus proyectos: abandonar el proyecto una vez iniciado, construir dicho proyecto por etapas, o expandir el mismo durante su ejecución o al término de éste.

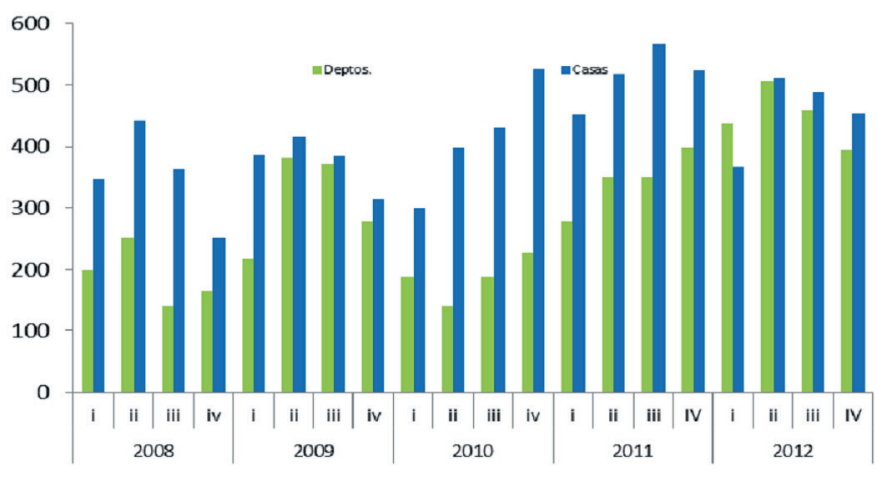

Figura 2: Venta trimestral de casas y departamentos en el Gran Concepción, desde el año 2008 al cuarto trimestre del 2012 (CChC, 2012)

\section{Caso de estudio para la aplicación de las opciones reales}

El proyecto de edificación que ha servido de referencia en esta investigación es un proyecto tipo en régimen de condominio, el que se ajusta a una amplia variedad de proyectos inmobiliarios en Chile, ya sea materializándolo de forma individual, o como parte de un proyecto más grande construido por etapas. Se trata de un condominio de 38 casas individuales de 50 a $75 \mathrm{~m}^{2}$, el cual se denominará Proyecto Casas. El proyecto consta de una estructura de costos e ingresos, además de la utilidad promedio sobre las ventas y sobre inversión (Forcael, 2012). Para la elección de la ubicación y el rango de precios de ventas del proyecto, se han considerado las proyecciones y estimaciones que otorga el Informe Inmobiliario del Gran Concepción de la Cámara Chilena de la Construcción (CChC, 2012).

El proyecto está emplazado en un lote de $5033 \mathrm{~m}^{2}$, con $2270 \mathrm{~m}^{2}$ construidos (considerando superficie útil más estacionamiento para cada casa), destinando para superficies comunes y áreas verdes $2763 \mathrm{~m}^{2}$. Es un proyecto rodeado de naturaleza, con cómodos espacios para habitar y disfrutar una vida en familia, con precios de venta que van desde las UF900 a UF1596. 
La primera opción a evaluar en el Proyecto Casas es la de abandonar el proyecto, ya que en todo desarrollo inmobiliario, una vez que se obtiene el permiso de edificación se comienza inmediatamente a vender, por lo que el comportamiento de éstas, durante su primer trimestre, indicaría la aceptación de las viviendas por el mercado. Por esta razón, se evaluará la opción de abandono, cuya inversión será de $I_{A}=\mathrm{UF1074}$, correspondiente al pago de los intereses del financiamiento externo más gastos generales de administración.

La segunda opción identificada en el proyecto, es la opción de expandir en el mismo terreno el número de casas en un $50 \%$ (19 casas) en el trimestre 5, ocupando la superficie que en un principio sería para las áreas verdes y comunes, sin financiamiento externo, invirtiendo $I_{E}=$ UF 10109.

\section{MBLT para la valoración de opciones reales}

Propuesto por Trigeorgis (1996), el Modelo Binomial Logarítmico Transformado MBLT corresponde a una variante numérica del modelo de valoración binomial, en el que se asume un contexto de valoración neutral al riego, con el cual se consigue aproximar, mediante un proceso de tipo discreto, la evolución del valor del proyecto o activo subyacente, que se supone es un período continuo. Además de ser un método numérico más consistente (considerando la misma media y varianza en todo momento) y más estable, presenta mejoras respecto al modelo binomial, ya que los errores de aproximación se reducen, siendo incluso más eficiente debido a la facilidad que ofrece para su utilización informática, por representar el proceso estocástico que sigue el activo subyacente.

La principal ventaja que presenta el MBLT respecto al método binomial original, es la de capturar las interacciones entre múltiples opciones presentes en los proyectos, representando, numéricamente, de manera más adecuada y apegada a la realidad la flexibilidad operativa de un proyecto, que para efectos de esta investigación es en el sector inmobiliario. Por lo tanto, este método permite evaluar opciones de manera eficiente, ya que considera las posibles interacciones entre éstas, realizando, así, una evaluación económica representativa de los proyectos de inversión.

Se define la variable $V$ que es el valor del activo subyacente; correspondiendo al valor presente obtenido a través del método tradicional del VAN, el cual no incorpora el valor de las opciones o las flexibilidades. Dentro de los supuestos del modelo, se considera que la variable $V$ tiene una distribución logarítmica-normal, y que su tasa de rendimiento continua se distribuye normalmente, siguiendo el proceso estocástico de difusión tal que:

$$
\frac{d V}{V}=\alpha d t+\sigma d z
$$

Donde $\alpha$ corresponde a la rentabilidad del proyecto, $\sigma$ es la desviación estándar instantánea de $V, \mathrm{y} d z$ es la diferencial de un proceso estándar de Wiener (con media 0 y varianza $d t$ ). En cualquier intervalo de tiempo $\Delta t$, la variable $X=\log$ $(V)$, seguiría un proceso Browniano aritmético, de manera que:

$$
\Delta X=\ln \left(\frac{V_{t+\Delta t}}{V_{t}}\right)=\left(r-\frac{\sigma^{2}}{2}\right) \Delta t+\sigma d z
$$

Los incrementos $\Delta X$ son idénticos, independientes $\mathrm{y}$ normalmente distribuidos con media $\mu=\left[R_{\mathrm{f}}-1 /\left(2 \sigma^{2}\right)\right] \Delta \mathrm{t} \mathrm{y}$ varianza $\sigma^{2} \Delta$ t. Si el proceso de difusión puede ser aproximado por un proceso idéntico en tiempo discreto, subdividiendo la vida total del proyecto $T$, en $N$ subintervalos de tiempo de longitud $\tau$, de modo que $T=\mathrm{N} \tau$ y $k=\frac{\sigma^{2}}{N} T$, entonces en cada subintervalo discreto $\tau, X$ seguirá un proceso estocástico Markoviano, es decir una sucesión de ensayos similares u observaciones en la cual cada ensayo tiene el mismo número finito de resultados posibles y en donde la probabilidad de cada resultado para un ensayo dado depende sólo del resultado del ensayo inmediatamente precedente y no de cualquier resultado previo. Con movimiento al alza en una cantidad $\Delta X=H$ con una probabilidad (neutral al riesgo) $P$, o un movimiento a la baja en una misma cantidad $\Delta X=-H$ con una probabilidad $1-P$. Además, la media y la varianza de este proceso discreto Markoviano, está dado por:

$\mathrm{E}(\Delta X)=2 P H-H \quad$ y $\operatorname{Var}(\Delta X)=H^{2}-[2 P H-H]^{2}$

$\mathrm{Al}$ igualarse con el otro proceso, según $\mu=2 P H-H$ y $k=$ $H^{2}-[2 P H-H]^{2}$ se obtienen los valores para $P$ y $H$ :

$$
P=\frac{1}{2}\left(1+\frac{\mu k}{H}\right) \quad \text { y } \quad H=\sqrt{k+(\mu k)^{2}}
$$




\section{Interfaces del algoritmo}

Para la primera interfaz se deben especificar los valores iniciales del modelo que afectan a las opciones como: $V$, $r, \sigma, T, N$ y la serie de inversiones relacionadas o precios de ejercicios de cada opción. La segunda interfaz incluye el cálculo, a partir de los parámetros de entrada indicados en el primer paso, de las variables necesarias para el desarrollo del algoritmo. En esta etapa se determinan: $K, \mu, H$ y $P$. En la tercera interfaz, corresponde el cálculo de los valores terminales en $j=N$, para cada estado $i$ del algoritmo, donde $j$ corresponde al número de intervalos de longitud $k$, a su vez $i$ denota el estado de la variable $X$ de modo que $X(i)=X_{0}+i H$. Por otro lado, $R(i)$ indica el valor total de la oportunidad de inversión en el estado $i$, determinando el valor del proyecto según:

$V_{i}=e^{X o+i H}$

Y el valor total de la oportunidad de inversión según $R^{\prime}(i)=\operatorname{Max}[V(i), 0]$.

Finalmente, la cuarta interfaz considera la resolución de un proceso iterativo hacia atrás, es posible obtener el VAN del proyecto sin opciones. Partiendo desde una condición terminal en $j=N$, analizando hacia atrás en un esquema triangular desde $j=N-1, \ldots, 1$. Para cada estado $i$ el algoritmo calcula el valor de la oportunidad de inversión, ocupando la información del intervalo anterior $j+1$. De esta forma, se va obteniendo el valor de la oportunidad de inversión de cada período $j$ en el estado $i$, según:

$R_{i}=e^{-\frac{r k}{\sigma^{2}}}\left[P R_{i+1}+(1-P) R_{i-1}\right]$

$\mathrm{Si}$ en cualquier instante de tiempo $j$, al realizar el movimiento iterativo hacia atrás, se encuentra una opción real, es necesario calcular el valor de la oportunidad de inversión de acuerdo a la Tabla 1.

Tabla 1: Ajustes del valor de la inversión según el tipo de opciones (Trigeorgis, 1996)

\begin{tabular}{lc}
\hline Tipo de Opción & Valor \\
\hline Opción de esperar un período & $R^{\prime}=\operatorname{Max}\left[e^{-\mathrm{r} \tau} E\left(R_{\mathrm{j}+1}\right), R_{\mathrm{j}}\right]$ \\
$\begin{array}{l}\text { Opción de abandonar, ahorrando } I_{1} \\
\text { Opción de contraer un c\%, ahorrando }\end{array}$ & $R^{\prime}=\operatorname{Max}\left(R-I_{1}, 0\right)$ \\
una parte $I_{2}$ ' de $I_{2}$ & $R^{\prime}=R+\operatorname{Max}\left(I_{2}{ }^{\prime}-\mathrm{cV}, 0\right)$ \\
$\begin{array}{l}\text { Opción de expandir un e\%, invirtiendo } I_{3} \\
\text { Opción de cambio a mejor uso alternativo }\end{array}$ & $R^{\prime}=R+\operatorname{Max}\left(e V-I_{3}, 0\right)$ \\
con valor de salvamento $S$ & $R^{\prime}=\operatorname{Max}(R, S)$ \\
\hline
\end{tabular}

Resultados

\section{Valoración de las opciones en el Proyecto Casas}

De acuerdo con los análisis efectuados, los parámetros de entrada para la aplicación del MBLT corresponden a los siguientes:

- Valor presente de los flujos de caja sin inversiones iniciales: UF8752, cuya estructura se presenta en la Figura 3.

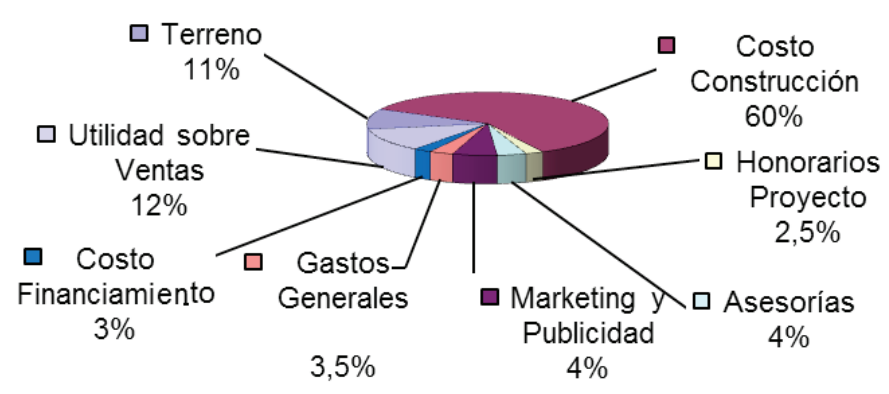

Figura 3: Estructura de costos y utilidades del proyecto en estudio.

- Volatilidad del proyecto: $\sigma=20.65 \%$

- Tasa libre de riesgo: $R_{\mathrm{f}}=6.35 \%$ (valor trimestral)

- Horizonte de evaluación del proyecto: 8 trimestres

- Opción de Abandono en el trimestre 2 (Opción put), invirtiendo $I_{\mathrm{A}}=\mathrm{UF} 1074$

- Opción de Expandir en un 50\% (Opción call) el número de casas en el trimestre 5. Invirtiendo $I_{\mathrm{E}}$ $=$ UF 10109

Donde el parámetro de volatilidad del proyecto se define como la medida de la oscilación que presenta una variable crítica del proyecto a través del tiempo, sobre la base de antecedentes históricos acerca de ésta (Mun, 2005). Al analizar el Mercado Inmobiliario del Gran Concepción, no se puede estar ajeno a los fenómenos como las crisis económicas, el terremoto del 27 de febrero del 2010 o las promociones y subsidios que son instancias que aceleran o desaceleran el crecimiento del sector. Un proyecto de inversión inmobiliaria (construcción de viviendas) está sujeto a diversas variables, las cuales afectan directamente los retornos esperados de éste. A éstas se pueden distinguir variables como el precio del suelo (por metro cuadrado) o el costo de construcción. Sin embargo el aumento progresivo 
en la escasez del suelo, considerando las actuales disposiciones que impiden la extensión del radio urbano, y la flexibilización de las zonificaciones existentes al interior de las comunas que considera el Gran Concepción, han elevado el valor del metro cuadrado de terreno y, por ende, el precio de las viviendas construidas. Por consiguiente, para efectos de este estudio, la volatilidad utilizada como parámetro de entrada del MBLT, se obtuvo a partir de los registros históricos del precio del suelo del Gran Concepción. Los datos fueron obtenidos de los Boletines del Mercado de Suelo Urbano del Gran Concepción elaborado por la Universidad del Bío-Bío (1989). Se consideraron los 89 Boletines que desde el cuarto trimestre de 1989 hasta el cuarto trimestre de 2011 se han publicado.

Con respecto a la tasa libre de riesgo, Aguilera (2011) menciona que a la hora de considerarla para proyectos inmobiliarios, se debe observar la tasa de interés de los Pagarés y Bonos licitados por el Banco Central de Chile (P.D.B.C.). Con el fin de automatizar la valoración del proyecto al trabajar con opciones reales, se utilizó una planilla de cálculo, la cual permitió valorar las opciones mediante el MBLT, considerando las variables tanto de entrada y las preliminares.
Para la correcta aplicación del método se deben considerar las cuatro etapas del MBLT. A partir de la información económica-financiera, se ingresan los parámetros de entrada, primera interfaz; los cuales afectan al proyecto y a las opciones reales que se han de evaluar. Valor presente de los flujos $\sin$ inversiones iniciales $V$, tasa de interés sin riesgo $R_{\mathrm{f}}$, volatilidad $\sigma$, horizonte temporal del proyecto $T$, número de intervalos $N$. Sucesivamente, como segunda interfaz, se deben calcular las variables preliminares del modelo $(k, \mu, H, P)$, ver Tabla 3 , las cuales permiten calcular la evolución del activo subyacente mediante:

Determinación de $k=\sigma^{2} T / N=0.0426$, la tendencia $\mu=R_{\mathrm{f}} / k$ $-1 / 2=0.9891$, evolución de la variable $\Delta X=H=0.2108$, la probabilidad de $\Delta \mathrm{X}=P=0.6001$ y $(1-P)=0.3999$, el operador de descuento para la valoración de opciones $\exp \left(-R_{\mathrm{f}} k / \sigma^{2}\right)=0.9385$.

En la Tabla 2 se muestra la evolución del proceso estocástico del valor del proyecto (segunda interfaz), realizado mediante la discretización del proceso en $T$ sub-intervalos de tiempo, considerando el alza y la baja del activo subyacente de acuerdo a las probabilidades $P$ y $(1-P)$.

Tabla 2: Evolución del proceso estocástico del valor del proyecto

\begin{tabular}{|c|c|c|c|c|c|c|c|c|c|}
\hline & 0 & 1 & 2 & 3 & 4 & 5 & 6 & 7 & 8 \\
\hline \multirow{2}{*}{$\begin{array}{l}X_{0}= \\
H_{0}=\end{array}$} & 9.0770 & & & & & & & & \\
\hline & 0.2108 & & & & & & & & \\
\hline & & & & & & & & & 10.7631 \\
\hline & & & & & & & & 10.5524 & \\
\hline & & & & & & & 10.3416 & & 10.3416 \\
\hline & & & & & & 10.1309 & & 10.1309 & \\
\hline & & & & & 9.9201 & & 9.9201 & & 9.9201 \\
\hline & & & & 9.7093 & & 9.7093 & & 9.7093 & \\
\hline & & & 9.4986 & & 9.4986 & & 9.4986 & & 9.4986 \\
\hline & & 9.2878 & & 9.2878 & & 9.2878 & & 9.2878 & \\
\hline & 9.0770 & & 9.0770 & & 9.0770 & & 9.0770 & & 9.0770 \\
\hline & & 8.8663 & & 8.8663 & & 8.8663 & & 8.8663 & \\
\hline & & & 8.6555 & & 8.6555 & & 8.6555 & & 8.6555 \\
\hline & & & & 8.4447 & & 8.4447 & & 8.4447 & \\
\hline & & & & & 8.2340 & & 8.2340 & & 8.2340 \\
\hline & & & & & & 8.0232 & & 8.0232 & \\
\hline & & & & & & & 7.8125 & & 7.8125 \\
\hline & & & & & & & & 7.6017 & \\
\hline & & & & & & & & & 7.3909 \\
\hline
\end{tabular}


De acuerdo a lo indicado, el software realizó una transformación logarítmica del valor presente de los flujos de caja del proyecto, sin considerar las inversiones iniciales, generando la variable $X_{0}$, según $X_{0}=\ln (V)=\ln$ $(8752)=9.077$. Luego, se generó la discretización del horizonte de evaluación del proyecto para $T=8$ e intervalos de largo $k=8$. Así, se modeló el proceso estocástico con alzas y bajas de magnitud $H_{0}$, con probabilidad $P$ y $1-P$, respectivamente.
Para la tercera interfaz del modelo, se determinan los valores iniciales en $j=N=8$, para cada estado $i$, donde $X_{i 8}$ $=e^{X_{i 8}}$. Una vez obtenidos los valores en la cuarta interfaz se calcula mediante un proceso iterativo hacia atrás para cada período $j(j=N, \ldots, 1)$ y para cada estado $i$, el valor del proyecto mediante un operador de descuento $\exp \left(-r k / \sigma^{2}\right)$, de acuerdo a (7).

Mediante esta interfaz se obtiene el VAN del proyecto sin opciones, mostrado en la Tabla 3.

Tabla 3: Valor del Proyecto Casas sin opciones

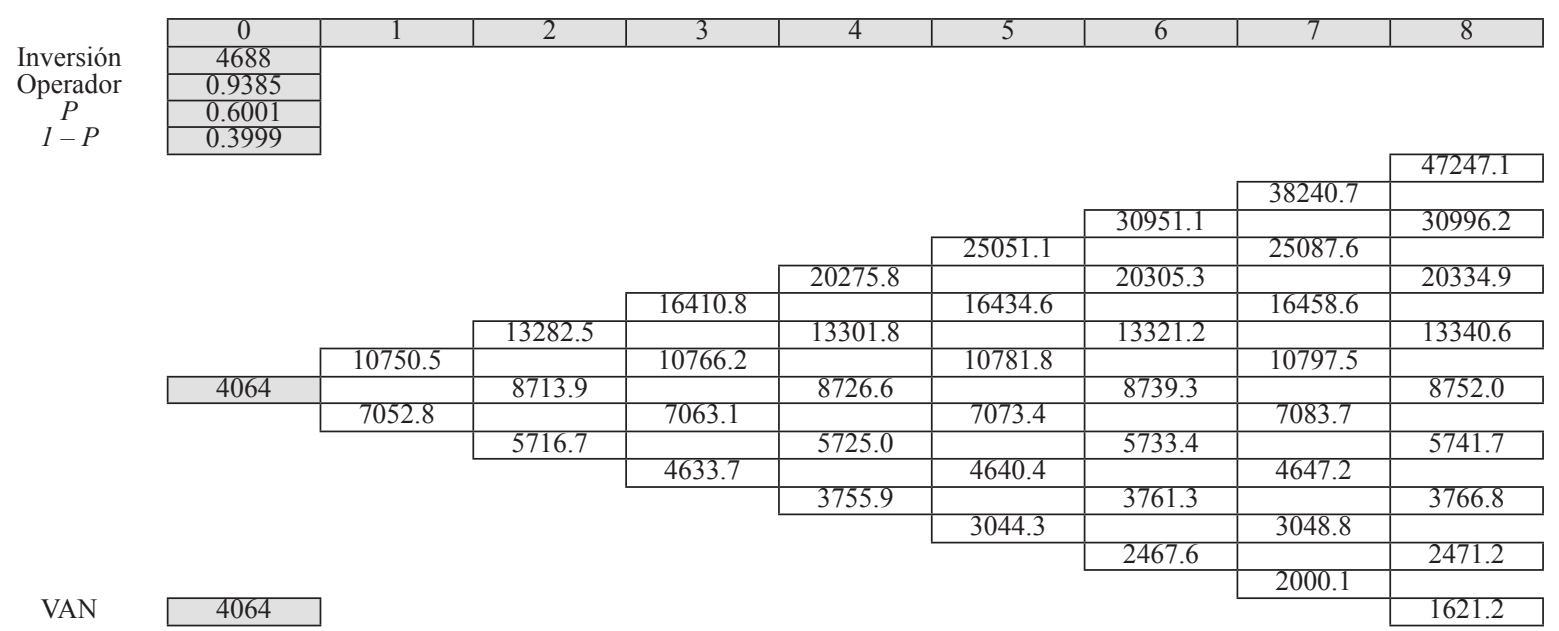

Al tener los resultados de las cuatro etapas del modelo y de las inversiones que se realizarían al abandonar y expandir el proyecto, se debe realizar el movimiento iterativo hacia atrás, ajustando el valor de la inversión según el tipo de opción Abandonar $=R^{\prime}=\operatorname{Max}\left(R-I_{1}, 0\right)$ y Expandir $=R^{\prime}=$
$R+\operatorname{Max}\left(\mathrm{eV}-I_{3}, 0\right)$. En la Tabla 4, se muestra el VAN Expandido del proyecto al considerar la primera opción identificada en el Proyecto Casas, Abandono, con una inversión $I_{A}=$ UF1074. Como la opción ha de ser ejercida en el trimestre 2 .

Tabla 4: Valor de la opción Abandono del Proyecto Casas

\begin{tabular}{|c|c|c|c|c|c|c|c|c|c|}
\hline \multirow{3}{*}{ Inversión } & & & & & & & & & \\
\hline & 0 & 1 & 2 & 3 & 4 & 5 & 6 & 7 & 8 \\
\hline & 4688 & & & & & & & & \\
\hline \multirow{15}{*}{$\begin{array}{c}\text { Operador } \\
P \\
1-P \\
\text { Opción de Abandono }\end{array}$} & 0.9385 & & & & & & & & \\
\hline & 0.6001 & & & & & & & & \\
\hline & 0.3999 & & & & & & & & 17247 \\
\hline & & & $10 / 4$ & & & & & 382407 & 47247.1 \\
\hline & & & & & & & 30951.1 & & 30996.2 \\
\hline & & & & & & 25051.1 & & 25087.6 & \\
\hline & & & & & 20275.8 & & 20305.3 & & 20334.9 \\
\hline & & & & 16410.8 & & 16434.6 & & 16458.6 & \\
\hline & & & 14356.5 & & 13301.8 & & 13321.2 & & 13340.6 \\
\hline & & 11758.5 & & 10766.2 & & 10781.8 & & 10797.5 & \\
\hline & 5010 & & 9787.9 & & 8726.6 & & 8739.3 & & 8752.0 \\
\hline & & 8060.7 & & 7063.1 & & 7073.4 & & 7083.7 & \\
\hline & & & 6790.7 & 46337 & 5725.0 & 46404 & 5733.4 & 46472 & 5741.7 \\
\hline & & & & & 3755.9 & & 3761.3 & & 3766.8 \\
\hline & & & & & & 3044.3 & & 3048.8 & \\
\hline \multirow{2}{*}{$\begin{array}{c}\text { Van } \\
\text { Van Expandido } \\
\text { Valor de la Opción }\end{array}$} & 4064 & & & & & & 2467.6 & & 2471.2 \\
\hline & $\begin{array}{c}5010.1 \\
946\end{array}$ & & & & & & & 2000.1 & 1621.2 \\
\hline
\end{tabular}


El VAN Expandido presentado en la Tabla 5 considera la segunda opción identificada en el Proyecto Casas,
Expandir el proyecto un 50\% (19 casas), con $e=50 \%$, una inversión $I_{E(5)}=$ UF 10109, ejercida en el trimestre 5.

Tabla 5: Valor de la opción Expandir del Proyecto Casas

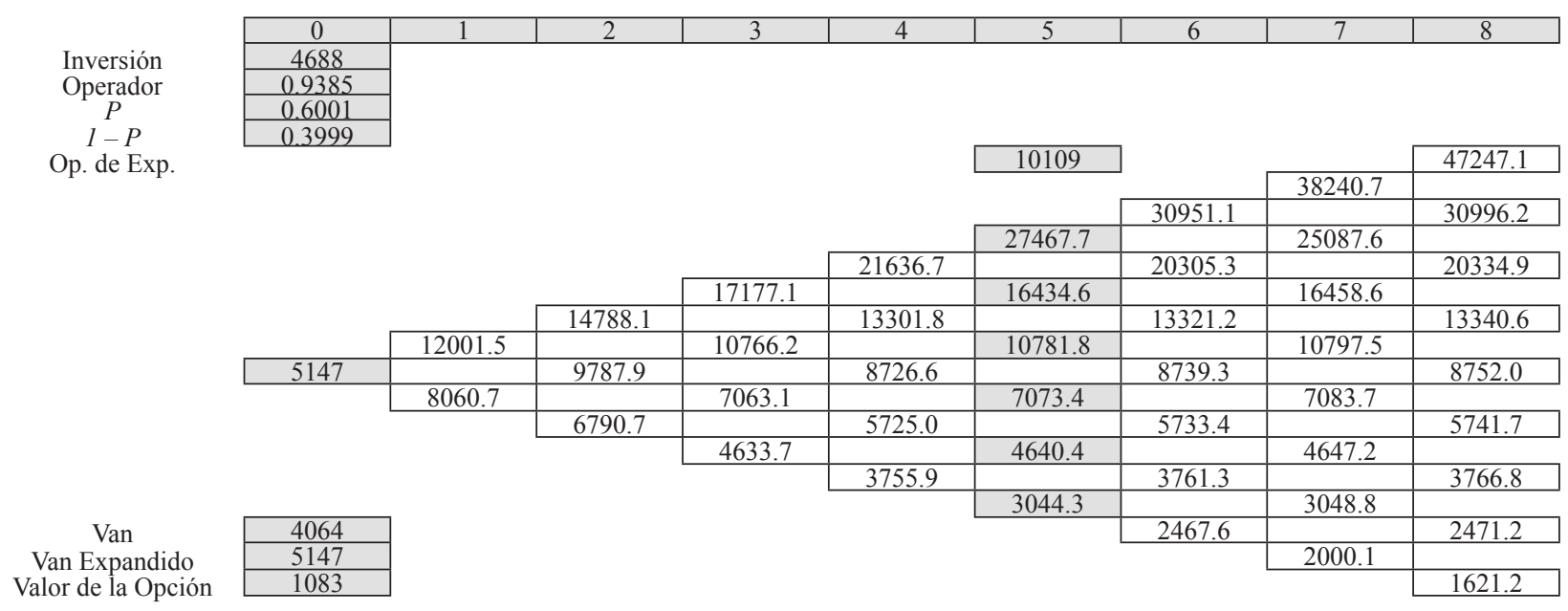

\section{Análisis de los resultados}

Con el método tradicional, la evaluación del Proyecto Casas dio como resultado un VAN de UF4064 y una TIR de un $13 \%$, descontado a una tasa de un $7.5 \%$ para un horizonte de evaluación de 8 trimestres. Las opciones reales consideradas en este proyecto, tanto el abandono como expandir en un 50\% el número de casas, responden a la incertidumbre dada en función del crecimiento de la población, el crecimiento económico e ingresos per cápita del Gran Concepción. El MBLT se basa, fundamentalmente, en considerar que el precio del activo subyacente (valor de los flujos de caja del proyecto $V$ ) sigue un proceso estocástico, el cual puede ser aproximado en tiempo discreto. Por lo tanto, se ha dividido la vida total del proyecto en $N=8$ subíntervalos de tiempo, por lo cual, el valor de la variable $X_{0}=\ln (V)$ ha seguido un proceso estocástico Markoviano con movimientos aleatorios ascendentes y descendentes; de acuerdo a la volatilidad presente en el activo subyacente y su tasa libre de riesgo, obteniendo así la evolución estocástica del proyecto. Este proceso ha quedado validado en el Proyecto Casas, ya que al calcular iterativamente el valor del proyecto, trayendo al presente el valor de los flujos descontados a la tasa libre de riesgo, ha dado como resultado el valor del VAN tradicional del proyecto (UF4064).

De acuerdo a la evolución estocástica del valor del proyecto, se ha podido aproximar el valor que tendría cada opción, al ser ejercida en el proyecto durante su periodo de evaluación. Contrastando los resultados del MBLT con los obtenidos a través del VAN tradicional, se puede comprobar que el Proyecto Casas presenta un potencial de agregación de valor mediante la ejecución de las opciones reales identificadas; utilizando como fuente de valor la incertidumbre.

La primera opción evaluada fue abandonar el proyecto en el trimestre 2. Como se observa en la Tabla 6, se obtiene un VAN Expandido de UF5010, agregando a la evaluación tradicional un valor de UF946 si se lleva a cabo la opción. La lógica radica en el hecho de construir 19 casas adicionales, considerando que en el trimestre 2 se habrían vendido las 38 casas, haciendo uso de la opción expandir, aprovechando el éxito del proyecto inicial y tomando en consideración el mismo terreno para edificar; en donde en un principio se tenían contempladas áreas verdes y espacios de recreación. 
Tabla 6: Resultados obtenidos a partir del análisis realizado con planilla de cálculo

\begin{tabular}{|c|c|c|c|}
\hline Opción & $\begin{array}{c}\text { VAN } \\
\text { tradicional }\end{array}$ & $\begin{array}{l}\text { Valor de la } \\
\text { opción }\end{array}$ & $\begin{array}{c}\text { VAN } \\
\text { Expandido }\end{array}$ \\
\hline Abandonar & UF4064 & UF946 & UF5010 \\
\hline Expandir & UF4064 & UF1083 & UF5147 \\
\hline $\begin{array}{l}\text { Opciones en } \\
\text { conjunto }\end{array}$ & UF4064 & UF2029 & UF6093 \\
\hline
\end{tabular}

La segunda opción real a ejercer fue expandir en un 50\% el número de casas (19 casas). Como se muestra en la Tabla 6, es la opción que tiene un mayor impacto en el valor del VAN del proyecto, ya que su VAN Expandido es de UF5147, otorgando un valor adicional de UF1083. La relevancia y la lógica de este resultado radica en que el proyecto se está expandiendo en un 50\%, de ahí se explica el mayor valor de la opción por sobre la de abandono.

Al comparar los resultados obtenidos con el método de las opciones reales y los calculados a través del VAN tradicional, se aprecia que al considerar en la evaluación la interacción de ambas opciones, el valor del proyecto es de UF6093; superior al valor calculado a partir del método tradicional, el cual alcanza un valor de UF4064. Esta diferencia ocurre debido a que las opciones de inversión capturadas por el método de las opciones reales aportan valor al proyecto. Para el Proyecto Casas las opciones presentes otorgan un valor de UF2029; representando la flexibilidad operativa del proyecto, o lo que es lo mismo, la capacidad de no seguir un único escenario de acción ante condiciones de entorno cambiantes, marcadas por la variabilidad del proceso.

Del mismo modo, se puede observar que el valor del proyecto calculado al considerar ambas opciones reales presentes en éste, es distinto del valor obtenido al realizar la suma individual de cada una de éstas (UF2029). Esto confirma el hecho de que utilizar un método de valoración que tenga en cuenta las interacciones entre opciones reales, tiene una importancia significativa en la evaluación del proyecto, al incorporar la incertidumbre en la evaluación. El hecho de expandir, traería un aumento en el beneficio del proyecto, ya que se venderían 19 casas más. Cabe destacar que la opción abandonar el proyecto en el trimestre 5 igual trae un beneficio para el inversionista, ya que éste deja de inyectar recursos a un proyecto del cual no se obtendrían beneficios. Por lo tanto, expandir el proyecto en un 50\% resulta ser la opción más recomendable a la hora de tomar una decisión. La opción de abandonar tiene mayor valor para los flujos de caja negativos, ya que permite evitar inversiones programadas ante escenarios desfavorables, controlando parcialmente las pérdidas.

Finalmente, al comparar los resultados obtenidos entre la evaluación tradicional del proyecto a través del VAN y el método de las opciones reales, se puede comprobar el potencial que tiene un proyecto que presenta cierta incertidumbre; corroborando el enfoque de Amram y Kulatilaka (2000), la incertidumbre crea oportunidades.

\section{Conclusiones}

Uno de los mayores problemas que se presenta al realizar el estudio y evaluación económica de proyectos, es el de justificar económicamente la inversión de recursos en un proyecto que se enfrenta a condiciones de alta volatilidad e incertidumbre. Esto se debe a que los flujos de caja obtenidos en la práctica, probablemente diferirán de los pronosticados por los evaluadores. Por consiguiente, al abordar nueva información producto del paso del tiempo, ocurriendo la disipación de la incertidumbre sobre las condiciones de mercado y los montos de los futuros flujos de caja, los evaluadores tendrán la flexibilidad de alterar la estrategia operativa inicialmente propuesta, con el objetivo de capitalizar las oportunidades de negocio que se presenten o para evitar y/o disminuir las pérdidas. La razón de ser de toda decisión que se tome para comenzar un proyecto de inversión, es que éste aporte el máximo de beneficios a largo plazo. Sin embargo, son muchas las opciones que se pueden tomar a lo largo de la vida del proyecto, las cuales no estaban consideradas en su evaluación inicial. Por lo tanto, un proyecto que valore y considere estas opciones valdría más que aquel que no lo haga.

En el caso del tipo de proyectos inmobiliarios considerados en este estudio, se presentan tres opciones reales: abandonar, aplazar y crecer por etapas, las cuales, si bien son consideradas por los participantes del sector de manera cualitativa, no logran mostrar su efecto en la evaluación. Por consiguiente, la aplicación de las opciones reales en este tipo de proyectos que presentan riesgos e incertidumbre, se ha convertido en una herramienta de apoyo para la evaluación tradicional. Es por esto que, a través de este estudio, se ha mostrado como una empresa puede recurrir al abandono del proyecto, en el caso de no seguir 
construyendo y también a expandir la obra. Al analizar los resultados calculados mediante el Método Binomial Logarítmico Transformado MBLT en el proyecto aplicado, se demostró que al considerar las opciones en la evaluación, el valor del proyecto fue superior al calculado mediante el método tradicional del VAN. Por esta razón, se puede concluir que el método de las opciones reales representa un valioso aporte para el proceso de evaluación y gestión estratégica de proyectos de inversión inmobiliaria, ya que los valores obtenidos al evaluar las opciones, representan la flexibilidad operativa del proyecto, es decir, de no seguir un escenario único, sino las posibilidades de acción ante entornos inciertos, marcadas por la variabilidad existente a lo largo del horizonte de evaluación del proyecto.

Hay que mencionar que las opciones reales, como todos los métodos de evaluación que últimamente se están estudiando y aplicando, pueden causar cierta resistencia al momento de considerarlas como metodología de evaluación para la toma de decisiones. Los métodos tradicionales como VAN y TIR, son los que se consideran, aun para evaluar los proyectos inmobiliarios, pero no incorporan la incertidumbre en su metodología. Sin embargo, se ha demostrado que las valoraciones con métodos alternativos, como las opciones reales, aportan importantes consideraciones de tipo cuantitativo a la evaluación. Por esta razón, es conveniente seguir desarrollando aplicaciones en este sector, y hacer que la incertidumbre se incluya cuantitativamente en la evaluación, con la finalidad de tomar decisiones de tipo estratégicas más eficientes.

\section{Referencias}

Aguilera, C. (2011). Tasa de descuento en proyectos inmobiliarios. [15 junio 2012] disponible en http://actualidadinmobiliariachile. blogspot.com/2011/07/la-tasa-de-escuento-en-proyectos.html

Amram, M. and Kulatilaka N. (2000). Real options: Managing strategic investment in an uncertain world. Financial management association survey and synthesis. Harvard Business School Press, Boston, Massachusetts.

Brealey, R., Marcus, A. y Myers, S. (2007). Fundamentos de Finanzas Corporativas. $5^{\circ}$ ed. Madrid, McGraw-Hill

Calle, A.M. y Tamayo, V.M. (2009). Decisiones de inversión a través de opciones reales. Estudios Gerenciales 25, N¹11, 107126
Campa, J.M. (1994). Decisiones de inversión bajo incertidumbre. Alta Dirección ES 30(175), 31-38

Capozza, D. and Li, Y. (1994). The intensity and timing of investment: the case of land. The American Economic Review 84, No.4, 889-904

CChC Cámara Chilena de la Construcción, (2012). Resumen Cifras Inmobiliarias. Unidad de Estudios de la Delegación Regional Concepción. Informe Inmobiliario del Gran Concepción de la CChC $4^{\circ}$ ed. 2(1), 13-27.

CChC Cámara Chilena de la Construcción (2012). Oferta y Venta Inmobiliaria del Gran Concepción. Unidad de Estudios de la Delegación Regional Concepción. Estadísticas Sectoriales Regionales y Nacionales 1(1), 17-18.

Fontaine, E. (1997). Evaluación Social de Proyectos. $11^{\circ} \mathrm{ed}$. Ediciones Universidad Católica de Chile.

Forcael, E. (2012). Evaluación Económica de Proyectos Inmobiliarios: Variables consideradas en la construcción de proyectos habitacionales. [Diciembre de 2012] disponible en www.forcael.cl

Hinojosa, S. (2008). Opciones Reales en Inversiones Públicas: Revisión de literatura, desarrollos conceptuales y aplicaciones. Tesis doctoral Escuela Superior de Administración y Dirección de Empresas ESADE, Barcelona

Lamothe, P. (1995). Opciones Financieras un Enfoque Fundamental. Madrid, España: McGraw-Hill

Majd, S. and Pindyck, R. (1987). Time to Build, Option Value, and Investment Decisions. Journal of Financial Economics 18(1), 7-27

Mascareñas, J. (2003). Opciones Reales y Valoración de Activos: Cómo Medir la Flexibilidad Operativa en la Empresa. $1^{\circ} \mathrm{ed}$. Madrid, Prentice Hall

Mun, J. (2005). Real Options Analysis: Tools and Techniques for Valuing Strategic Investment and Decisions. 2nd Edition, Wiley Finance

Quigg, L. (1993). Empirical Testing of Real Options - Pricing Models. Journal of Finance 1(1), 621-640

Sánchez, Y. y Andalaft, A. (2010). Evaluación de inversiones forestales mediante opciones reales. Ingenerare 24, 41-49

Sapag, N. y Sapag, R. (2008). Preparación y Evaluación de Proyectos. $5^{\text {ta }}$ ed. Santiago, McGraw-Hill 
Titman, S. (1985). Urban land prices under uncertainty. The American Economic Review 3(75), 505-514

Trigeorgis, L. (1996). Real Options, Managerial Flexibility and Strategy in Resource Allocation. MIT Press, Cambridge, MA.

Trigeorgis, L. and Mason, S.P. (1987). Valuing managerial flexibility. Midland Corporate Finance Journal 5(1), 14-21
Universidad del Bío-Bío. (1989). Mercado de Suelo Urbano en el Gran Concepción. Vol. 1-89, 10-14.

Williams, J. (1991). Real Estate Development as an Option. Journal of Real Estate Finance and Economics 2(4), 191-208 